\title{
Children with significant cervical lymphadenopathy: clinicopathological analysis and role of fine-needle aspiration in Indian setup
}

\author{
Rizwan A. Khan, ${ }^{1}$ Shagufta Wahab, ${ }^{2}$ R. S. Chana, ${ }^{3}$ S. Naseem, ${ }^{4}$ S. Siddique ${ }^{5}$
}

\begin{abstract}
Objective: To study the clinicopathological profile of children from India with cervical lymphadenopathy and the role of fine-needle aspiration cytology with special emphasis on tuberculosis as a cause.

Methods: A total of 89 children in the age group of 10 months to 12 years, presenting to our hospital from April 2004 to March 2005, were included. All the patients underwent thorough clinical and investigational assessment vis-à-vis cervical lymphadenopathy. Outcome measurements included clinical status and ability of conventional tests to categorize different types of lymphadenopathy and their utility in diagnosing tubercular lymphadenitis. Interobserver variability was analyzed measuring kappa test and was found to be in agreement.
\end{abstract}

Results: Reactive hyperplasia was the most common type of lymphadenitis, followed by granulomatous involvement. Unilateral posterior triangle lymph nodes were the most commonly affected in the tubercular cervical lymphadenopathy group. Fine-needle aspiration followed by Ziehl-Neelsen staining, histopathology and culture in combination were able to perform the diagnosis in $85.7 \%$ of cases affected with tubercular etiology.

Conclusions: Fine-needle aspiration is a valuable diagnostic tool in the management of children with the clinical presentation of enlarged cervical lymph nodes. The technique reduces the need for more invasive and costly procedures, especially in a Third World country. Culture and histopathology, however, should be considered in cases where repeated fine-needle aspiration cytology is non-diagnostic.

J Pediatr (Rio J). 2008;84(5):449-454: Cervical lymphadenopathy, fine-needle aspiration cytology, children.

\section{Introduction}

In a typical outpatient pediatric clinic of a hospital in India, children with enlarged cervical, axillary or inguinal lymph nodes are a common presentation. ${ }^{1}$ The condition most commonly represents a transient response to a benign local or generalized infection, but occasionally it might herald the presence of a more serious disorder. Subacute or chronic lymphadenopathy is a manifestation of a number of diseases, such as tuberculosis, leprosy, mycoses, sarcoidosis, lymphoma and metastatic lymphadenopathy. With prevalence as high as $1.5 \%,{ }^{2}$ tuberculosis is still rampant in this part of the world and the tuberculous involvement of lymph nodes is the most common form of extrapulmonary tuberculosis (responsible for $30-40 \%$ of cases). ${ }^{3}$ Therefore, on initial examination tuberculosis is attributed as the underlying cause in most cases of lymphadenopathy. ${ }^{4,5}$ Tuberculous involvement of

1. MCh. Lecturer, Division of Paediatric Surgery, Department of Surgery, Jawaharlal Nehru Medical College Hospital (JNMCH), Aligarh Muslim University (AMU), Aligarh, India.

2. MD. Lecturer, Department of Radiodiagnosis, JNMCH, AMU, Aligarh, India.

3. MCh. Professor, Division of Pediatric Surgery, Department of Surgery, JNMCH, AMU, Aligarh, India.

4. MD. Senior resident, Department of Pathology, JNMCH, AMU, Aligarh, India.

5. MD. Lecturer, Department of Pediatrics, JNMCH, AMU, Aligarh, India.

No conflicts of interest declared concerning the publication of this article.

Suggested citation: Khan RA, Wahab S, Chana RS, Naseem S, Siddique S. Children with significant cervical lymphadenopathy: clinicopathological analysis and role of fine-needle aspiration in Indian setup. J Pediatr (Rio J). 2008;84(5):449-454.

Manuscript received Apr 22 2008, accepted for publication Aug 202008.

doi:10.2223/JPED. 1840 
superficial lymph nodes is common presentation of extrapulmonary tuberculosis in children. ${ }^{6,7}$ Lymphadenitis may be the only manifestation of the disease and there may not be associated constitutional symptoms, such as low grade fever, loss of weight, cough or other respiratory symptoms. ${ }^{8}$

Fine-needle aspiration cytology (FNAC) is an easy, rapid, economic and less invasive diagnostic modality for evaluating lymphadenitis. ${ }^{9,10}$ Diagnosis of tuberculosis in lymph nodes can be established by demonstrating acid-fast bacilli (AFB) in FNA smears with Ziehl-Neelsen stain or auramine-rhodamine stain, mycobacterial culture or through amplification of bacterial DNA by polymerase chain reaction (PCR). ${ }^{9}$ However, in India, being a developing country, the logistics (cost, equipment and time) involved in other techniques are too much; therefore, demonstration of AFB by Ziehl-Neelsen staining in FNAC smears is the most widely used technique. In addition, FNAC is a rapid, simple and cost-effective diagnostic technique for diagnosing reactive hyperplasia, infections, granulomatous lymphadenopathies, and metastatic diseases. ${ }^{11,12}$ The aim of the present study is to evaluate the role of FNA in categorizing different types of lymphadenopathy in children and its role in correlating cytological findings with clinical diagnosis.

\section{Methods}

A total of 89 patients under 12 years of age who presented to the Outpatient Department of Pediatric Medicine and Pediatric Surgery in JN Medical College, Aligarh, between April 2004 and March 2005 with main complaints of enlarged cervical lymph nodes were included in the study. The study was approved by the ethics committee. Inclusion criteria were: children $<12$ years with or without history of constitutional symptoms of tuberculosis, such as low grade fever, on and off cough with sputum, loss of appetite, and loss of weight; cervical lymph nodes $>1 \mathrm{~cm}$, which were matted on palpation without signs of inflammation; and duration of complaints for more than 4 weeks. Patients who were older than 12 years, systemically ill and required admission and patients with florid signs of inflammation or frank abscess in cervical region were excluded from the study. Each patient was thoroughly examined. A detailed history and physical examination was carried out and data regarding age, sex, lymph nodes involved, matted or discrete, history of contact with a diagnosed case of tuberculosis, relationship with the case, previous treatment for tuberculosis and presenting complaints were recorded.

Complete blood count and Mantoux test was performed in each case. Mantoux was taken as positive if there was an area of induration with transverse diameter $\geq 10 \times 10 \mathrm{~mm}$. FNAC, using a 25 -gauge needle and a 10 -mL syringe was performed using standard technique. FNAC was performed on the cases by an experienced pathologist as an outpatient procedure. After recording the gross appearance of the aspirate, drops of the aspirate material were immediately expressed and spread on glass slides, fixed with alcohol, and stained with hematoxylin and eosin, and Ziehl-Neelsen stain. Smears and tissue sections were stained with hematoxylin and eosin, and Ziehl-Neelsen stain and screened by an experienced pathologist. Results were recorded as suggestive of tuberculosis if there was typical caseating necrosis in the absence of AFB or positive if AFB were detected by Ziehl-Neelsen stain. If the AFB was negative in one slide, then another slide was stained and examined before labeling the case as negative.

Specimens from lymph node aspirates were processed and concentrated by centrifugation. The sediment was then inoculated onto two slants of Lowenstein-Jensen medium and incubated at $37^{\circ} \mathrm{C}$. The slants were examined weekly for 8 weeks for the visible appearance of colonies. Identification of mycobacteria was based on colony morphology, colony pigmentation, growth rate on conventional solid media. On the day of detection, the media were examined by Ziehl-Neelsen staining to determine whether they contained AFB or contaminants.

\section{Results}

A total of 89 patients participated in the study. Tuberculosis was the cause of cervical lymphadenitis in 35 patients. Other causes of cervical lymphadenopathy were reactive hyperplasia in 49 patients and lymphoma in five patients. The median age of these children, of whom 40 were boys, was 49 months (ranging from 10 to 141 months). There was a slight female preponderance as the male to female ratio was $1: 1.22$.

Of the 89 patients, 38 were clinically suspected to be suffering from tubercular involvement of cervical lymph nodes and were designated as the tubercular cervical lymphadenopathy (TCL) group. Five patients had a history of contact with a diagnosed case of tuberculosis. Two of our patients had a history of contact in the neighborhood, while three had a history of contact with a close family member (grandparents and father). None of the patients had previous history of the disease. The presenting complaints are summarized in Table 1. Unilateral posterior triangle involvement was the most common, seen in $48 \%$ of the cases. Pattern of lymph node involvement is depicted in Figure 1. Thirty-one had lymph node $>$ $1.5 \mathrm{~cm}$, while the other seven had lymph nodes between 1.0 and $1.5 \mathrm{~cm}$. Lymph nodes were matted in 20 patients. Mantoux test was in positive range in 20 patients. Complete blood count of 19 patients revealed predominance of lymphocytes, while 12 patients showed increased polymorphonuclear (PMN) counts.

All 38 patients in the TCL group were subjected to FNAC. In seven patients FNAC was suggestive of reactive hyperplasia, and one patient showed features of lymphoma, which was subsequently confirmed on biopsy and histopathology. The remaining 30 patients showed granulomatous disease characterized by aggregates of epithelioid cells with elongated nuclei and pale cytoplasm with occasional giant cells. 
Table 1 - Symptoms in patients with tuberculous cervical lymphadenitis

\begin{tabular}{lc}
\hline Symptoms & Number of patients (\%) \\
\hline Neck swelling (s) & $38(100)$ \\
History of contact & $9(23.6)$ \\
Weight loss & $6(15.7)$ \\
Fever & $4(10.5)$ \\
Cough & $4(10.5)$ \\
\hline
\end{tabular}

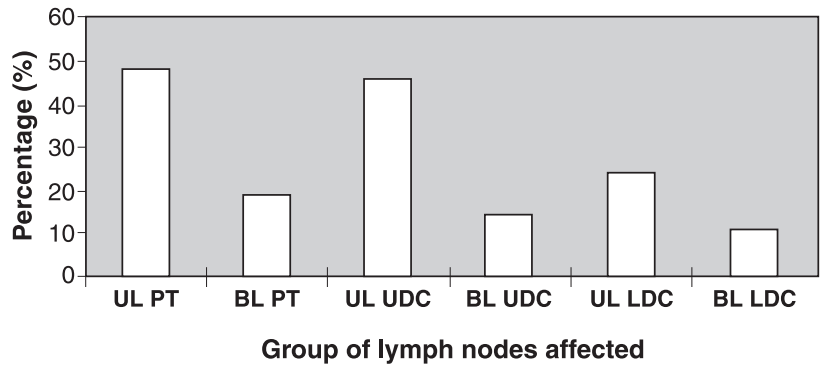

FNAC = fine-needle aspiration cytology; $\mathrm{HP}=$ histopathology.

Figure 1 - Pattern of involvement of different group of lymph nodes

Twenty-one of these 30 patients were positive for AFB, and 17 patients also showed caseous necrosis. Nine patients showed only granulomatous involvement and Ziehl-Neelsen staining for AFB was repeatedly (twice) negative. Therefore, these patients were subjected to culture and histopathological examination. Four patients turned out to be positive for tuberculosis (three were positive on histopathology and one required culture for confirmation) (Table 2 ). In the remaining five patients nothing conclusive for tuberculosis was found except for granulomatous involvement.

The non-tuberculous cervical Iymphadenopathy (non-TCL) group had 51 patients. Clinically lymph nodes were between $1.0-1.5 \mathrm{~cm}$ in 41 patients, while the other 10 patients had lymph nodes $>1.5 \mathrm{~cm}$. On palpation they were mostly ( $\mathrm{n}$ $=38,74.5 \%$ ) discrete. The most common group of lymph nodes affected in this group was submandibular (seen in $67 \%$ of cases) followed by submental group of lymph nodes. There was predominance of polymorphs in complete blood counts in $92 \%$ of the patients. FNAC of these patients revealed that 42 had reactive hyperplasia, five had granulomatous involvement of the lymph nodes, four had findings suggestive of lymphoma, which was later confirmed on biopsy and histopathological examination. Of the five patients who showed granulomatous disease, smears of three patients were positive for AFB on Ziehl-Neelsen staining, while one required culture and one needed histopathology for confirmation (Table 2).

Thus, 30 patients had their diagnosis confirmed by various combinations of the tests. Five patients were empirically put on antitubercular drug therapy based on the prevalence, clinical history and examination findings and on the fact that they showed granulomas on smear and histopathology examination, although no definite evidence of tubercle bacilli could be found. These patients also showed adequate response in the immediate follow-up and were put on short-course anti-tubercular treatment along with the other patients with confirmed diagnosis. Of the 35 cases of TCL, 24 (68.5\%) patients were diagnosed by demonstrating Ziehl-Neelsen staining for AFB following FNAC. Histopathological examination and subsequent staining with Ziehl-Neelsen stain was required to confirm the diagnosis in $11.4 \%$ of the cases ( $n=$ 4). Culture was required to confirm the diagnosis in $5.7 \%$ of cases $(n=2)$ (Figure 2 ).

\section{Discussion}

Cervical lymphadenopathy forms a common clinical problem in the pediatric age group.

Table 2 - Acid-fast bacilli results in the two subgroups

\begin{tabular}{lcccc}
\hline & \multicolumn{4}{c}{ Positive on the basis of } \\
\cline { 2 - 4 } Clinical type & FNAC & HP & Culture & Total \\
\hline TCL group $(n=38)$ & 21 & 3 & 1 & 25 \\
Non-TCL group $(n=51)$ & 3 & 1 & 1 & 5 \\
Total & 24 & 4 & 2 & 30
\end{tabular}

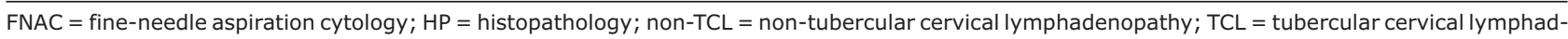
enopathy. 


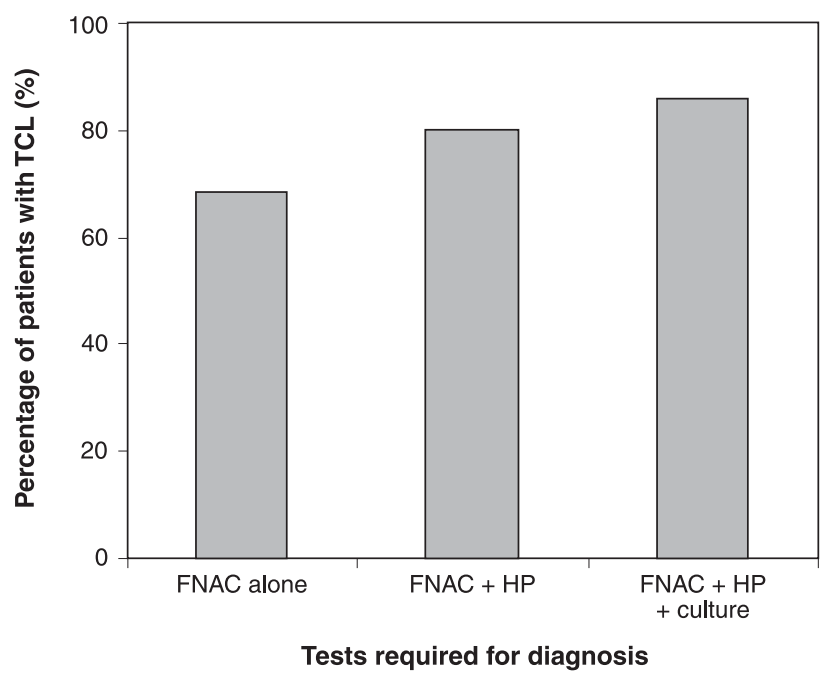

FNAC = fine-needle aspiration cytology; HP = histopathology.

Figure 2 - Percentage of cases diagnosed by different combinations of tests

In childhood enlargement of peripheral lymph nodes is most commonly caused by a local inflammatory process with viral upper respiratory tract infection or streptococcal pharyngitis as the common cause. ${ }^{4,13}$ In developing countries children having chronic cervical lymphadenopathy with firm, enlarged, painless lymph nodes, which are matted together, have a high incidence of infective causes, including a significant incidence of Mycobacterium tuberculosis and need a more detailed diagnostic evaluation in order to exclude malignant or granulomatous diseases. ${ }^{13,14}$

Tuberculosis is still one of the leading health problems in developing countries, with vast social and massive economic implications. In addition, it has shown resurgence in developed countries due to high incidence of HIV in these countries. ${ }^{15}$ In India about $1.5 \%$ of the population is affected with tuberculosis. $^{2}$ Tuberculous lymphadenitis is the most common presentation of extrapulmonary tuberculosis, accounting for $30-40 \%$ of cases in reported series. ${ }^{3}$ The onset of tuberculous lymphadenitis is insidious. Patients present with enlarged, usually painless lymph nodes, most commonly affecting the cervical region. Systemic symptoms such as fever, weight loss, and malaise may or may not be present at an early stage. Advanced disease presents additionally with cold abscess with or without sinus formation. Prompt diagnosis and effective treatment lead to reduced morbidity and mortality. It has been found that females are more likely to suffer from the disease. ${ }^{16,17}$ This was also found in our study with male to female ratio of $1: 1.22$. The cause may be women's underprivileged condition in the society in many parts of rural India.

There has been a definite change in clinical pattern, mainly because of nonspecific and irregular treatments before the diagnosis. ${ }^{18}$ In our study, most of the patients did not have constitutional symptoms. The most common nodal group affected was the unilateral posterior triangle group. The number of patients with matting was quite significant $(n=20)$. Similar results have also been reported in other studies. ${ }^{19,20}$ FNAC is a well established diagnostic tool in the assessment of cervical masses. In developing countries where tuberculous infection is common and other granulomatous diseases are rare, presence of granulomatous features on FNAC is highly suggestive of tuberculosis. ${ }^{21}$ In our study we found it to be a very useful diagnostic tool to identify patients with tuberculous lymphadenopathy. It obviates the need for excisional biopsy in most patients. Similar views have been expressed by others. ${ }^{22}$ The Mantoux test was a useful adjunct to the diagnosis. The most reliable indicator of cervical mycobacterial infection is an acid-fast smear from the FNA specimen. ${ }^{23}$

In our study we were able to diagnose $68.5 \%$ of cervical lymphadenopathy cases based on Ziehl-Neelsen staining following FNAC of the enlarged lymph nodes. When we combined the histopathological and culture to the tests, diagnosis was achieved in $85.7 \%$ of the cases. It can therefore be concluded that FNAC is a reliable diagnostic tool in helping to avert the more invasive surgical procedures undertaken in the diagnosis of tuberculous adenitis. The Ziehl-Neelsen stain for identification of AFB should be incorporated as an adjunct to increase the diagnostic accuracy of tuberculous lymphadenitis. ${ }^{24}$

Despite its well-established usefulness in the diagnosis of cervical tuberculous lymphadenitis, FNAC has several limitations in its clinical applications. Since it requires $>10,000$ organisms $/ \mathrm{mL}$, it cannot be used to distinguish between various members of mycobacteria, and its sensitivity ranges between $10-50 \%$ depending on the study. In this case, excisional biopsy and subjecting the aspirate for cytological examination is a very useful tool for the diagnosis of cervical tuberculous lymphadenitis. Some studies have suggested use of PCR in diagnosing these remainder cases of tuberculosis. ${ }^{25-27}$

The role of PCR in the detection of mycobacteria is not foolproof because its sensitivity for the detection of mycobacteria has been reported to range from 55 to $100 \% .{ }^{28-31}$ On the other hand, there is certainly a role of this investigation when the bacterial load is very low. ${ }^{15,32}$ However, in a developing country like India the cost associated with such investigations could lead to the likely patient's aversion to such costly investigation modality and treatment thereafter. To maximize the success rate of diagnoses, a combination of culture, cytological examination of FNA samples, supplemented with tuberculin skin testing seems to be the most effective strategy.

Thus, cervical lymph node enlargement is a common clinical problem in the pediatric population, reactive and granulomatous enlargement being two important causes. Firm, 
enlarged, painless lymph nodes which are matted together and fixed to the skin or underlying tissues require a more detailed diagnostic evaluation. Primary diagnostic evaluation of childhood peripheral lymphadenopathy is mainly based on group of lymph nodes involved and FNA. A careful history and thorough physical examination are the first steps in establishing the cause of a neck mass. Location, size, consistency, and mobility of the mass provide clues and are useful for comparison during follow-up. Our experience indicates that FNA is a rapid, simple, accurate diagnostic procedure and important initial step in the evaluation and management of enlarged cervical lymph nodes in children. It is very well tolerated by children, and there were no complications.

\section{References}

1. Bedi RS, Thind GS, Arora VK. A clinical-pathological study of superficial lymphadenopathy in Northern India. Indian J Tuberc. 1987;34:189-91.

2. Mukherjee AK. Tuberculosis control programme in India: progress and prospects. Indian J Tuberc. 1995;42:75-85.

3. Hooper AA. Tuberculous peripheral lymphadenitis. Br J Surg. 1972;59:353-9.

4. Leung AK, Robson WL. Childhood cervical lymphadenopathy. J Pediatr Health Care. 2004;18:3-7.

5. Talib VH, Pandey J, Khurana SK. Tuberculosis: an epidemic in the making. Ind J. Pathol Microbiol. 1993;36:339-40.

6. Narang P, Narang R, Mendiratta DK, Sharma SM, Narang R, Nayar S. Field study to evaluate the bacteriological parameters in the diagnosis of lymphnode tuberculosis in children. Indian J Tuberc. 1998;45:211-4.

7. Aggarwal P, Wali JP, Singh S, Handa R, Wig N, Biswas A. A clinico-bacteriological study of peripheral tuberculous lymphadenitis. J Assoc Physicians India. 2001;49:808-12.

8. Seth V, Kabra SK, Jain Y, Semwal OP, Mukhopadhyaya S, Jensen RL. Tubercular lymphadenitis: Clinical manifestations. Indian J Pediatr. 1995;62:565-70.

9. Raviglione MC, O'Brien RJ. Tuberculosis. In: Fauci AS, Braunwald E, Isselbacher KJ, Wilson JD, Martin JB, Kasper DL, et al., editors. Harisson's principles of internal medicine. 14th ed. New York: McGraw-Hill; 1998. p.1004-14.

10. van de Schoot $L$, Aronson DC, Behrendt $H$, Bras J. The role of fine-needle aspiration cytology in children with persistent or suspicious lymphadenopathy. J Pediatr Surg. 2001;36:7-11.

11. Prasoon D. Acid-fast bacilli in fine needle aspiration smears from tuberculous lymph nodes. Acta Cytologica. 2000;44:297-300.

12. Gupta RK, Naran S, Lallu S, Fauck R. The diagnostic value of fine needle aspiration cytology (FNAC) in the assessment of palpable supraclavicular lymph nodes: a study of 218 cases. Cytopathology. 2003;14:201-7.
13. Benesch $M$, Kerbl R, Wirnsberger A, Stunzner $D$, Mangge $H$, Schenkeli R, et al. Peripheral lymphadenopathy in childhood recommendations for diagnostic evaluation. Klin Padiatr. 2000; 212:277-82.

14. Moore SW, Schneider JW, Schaaf HS. Diagnostic aspects of cervical lymphadenopathy in children in the developing world: a study of 1,877 surgical specimens. Pediatr Surg Int. 2003;19: 240-4.

15. Geldmacher H, Taube C, Kroeger C, Magnussen H, Kirsten DK. Assessment of lymph node tuberculosis in northern Germany: a clinical review. Chest. 2002;121:1177-82.

16. Dandapat MC, Mishra BM, Dash SP, Kar PK. Peripheral lymph node tuberculosis: a review of 80 cases. Br J Surg. 1990;77: 911-2.

17. Subrahmanyam M. Role of surgery and chemotherapy for peripheral lymph node tuberculosis. Br J Surg. 1993;80:1547-8.

18. Jha BC, Dass A, Nagarkar NM, Gupta R, Singhal S. Cervical tuberculous lymphadenopathy: changing clinical pattern and concepts in management. Postgrad Med J. 2001;77:185-7.

19. Cheung WL, Siu KF, Ng A. Tuberculous cervical abscess: comparing the result of total excision against simple incision and drainage. $\mathrm{Br}$ J Surg. 1988;75:563-4.

20. Baskota DK, Prasad R, Kumar Sinha B, Amatya RC. Distribution of lymph nodes in the neck in cases of tuberculous cervical lymphadenitis. Acta Otolaryngol. 2004;124:1095-8.

21. Lau SK, Wei WI, Hsu C, Engzell UC. Efficacy of fine needle aspiration cytology in the diagnosis of tuberculous cervical lymphadenopathy. J Laryngol Otol. 1990;104:24-7.

22. Lau SK, Wei WI, Kwan S, Yew WW. Combined use of fine-needle aspiration cytologic examination and tuberculin skin test in the diagnosis of cervical tuberculous lymphadenitis. A prospective study. Arch Otolaryngol Head Neck Surg. 1991;117:87-90.

23. Weiler Z, Nelly P, Baruchin AM, Oren S. Diagnosis and treatment of cervical tuberculous lymphadenitis. J Oral Maxillofac Surg. 2000;58:477-81.

24. Bezabih M, Mariam DW, Selassie SG. Fine needle aspiration cytology of suspected tuberculous lymphadenitis. Cytopathology. 2002; 13:284-90.

25. Baek CH, Kim SI, Ko YH, Chu KC. Polymerase chain reaction detection of Mycobacterium tuberculosis from fine-needle aspirate for the diagnosis of cervical tuberculous lymphadenitis. Laryngoscope. 2000;110:30-4.

26. Singh KK, Muralidhar M, Kumar A, Chattopadhyaya TK, Kapila K, Singh MK, et al. Comparison of in house polymerase chain reaction with conventional techniques for the detection of Mycobacterium tuberculosis DNA in granulomatous lymphadenopathy. J Clin Pathol. 2000;53:355-61.

27. Huhti E, Brander E, Paloheimo S, Sutinen S. Tuberculosis of the cervical lymph nodes: a clinical, pathological and bacteriological study. Tubercle. 1975;56:27-36.

28. Narita M, Shibata M, Togashi T, Kobayashi H. Polymerase chain reaction for detection of mycobacterium tuberculosis. Acta Paediatr. 1992;81:141-4.

29. Shankar P, Manjunath N, Lakshmi R, Aditi B, Seth P, Shriniwas. Identification of Mycobacterium tuberculosis by polymerase chain reaction. Lancet. 1990;335:423.

30. >Brisson-Noël A, Gicquel B, Lecossier D, Lévy-Frébault V, Nassif $X$, Hance AJ. Rapid diagnosis of tuberculosis by amplification of mycobacterial DNA in clinical samples. Lancet. 1989;2:1069-71. 
31. Del Prete R, Mosca A, D'Alagni M, Sabato R, Picca V, Miragliotta G. Detection of Mycobacterium tuberculosis DNA in blood of patients with acute pulmonary tuberculosis by polymerase chain reaction and non-isotopic hybridization assay. J Med Microbiol. 1997;46:495-500.

32. Mirza S, Restrepo BI, McCormick JB, Fisher-Hoch SP. Diagnosis of tuberculosis lymphadenitis using a polymerase chain reaction on peripheral blood mononuclear cells. Am J Trop Med Hyg. 2003; 69:461-5
Correspondence:

Rizwan Ahmad Khan

4/817-F, S. S. Nagar

U.P. 202002 - Nagla Road, Aligarh - India

Tel.: +91 (092) 1697.1976

E-mail: drrizwanahmadkhan@yahoo.co.in 